\title{
Circumstellar medium around close southern PMS binaries ${ }^{\star}$
}

\section{New results with the ESO ADONIS + COMIC/SHARP infrared system}

\author{
H. Geoffray ${ }^{1,3}$ and J.-L. Monin ${ }^{1,2}$ \\ 1 Laboratoire d'Astrophysique, Observatoire de Grenoble, Université Joseph Fourier, BP 53, \\ 38041 Grenoble Cedex, France \\ 2 Institut Universitaire de France \\ 3 CNES, 18 Av. E. Belin, 31400 Toulouse, France \\ e-mail: Herve.Geoffray@cnes.fr
}

Received 12 December 2000 / Accepted 19 January 2001

\begin{abstract}
We have started a series of high angular resolution observations of close southern pre Main-Sequence (PMS) binary systems in order to study their respective photometry from 1 to $5 \mu \mathrm{m}$. For most of the binaries of our sample, we present the first near infrared (NIR) measurements longward of $3 \mu \mathrm{m}$ on both components. From these measurements we characterize the stellar Spectral Energy Distribution (SED) of the primary and its companion, adjust standard photospheres to estimate their luminosities \& temperatures, hence masses \& ages through the use of an HR diagram. At the same time, we study the presence of circumstellar material around our PMS targets, in order to assess how the eventually leftover material is distributed after contraction. We find the same proportion of mixed systems (CTTS/WTTS) as in the overall southern clouds and all these mixed systems are found with mass ratios $<0.5$; all the WTTS/WTTS systems of our sample are found in binaries closer than $60 \mathrm{AU}$; apart from one of the most separated binaries, all our objects appear coeval.
\end{abstract}

Key words. stars: binaries: close - circumstellar matter - stars: formation - infrared: stars - instrumentation: adaptive optics - techniques: photometric

\section{Introduction}

The process of low-mass star formation is now well known for producing a large fraction of binary and multiple systems. This result is confirmed by many surveys (e.g. Reipurth \& Zinnecker 1993; Ghez et al. 1993; Leinert et al. 1993), showing that most if not all T Tauri stars (TTS) have companion(s) (see e.g. Duchene 1999 for a review of the binary excess in star forming regions). Therefore, the study of PMS binary systems appears as a crucial key to understand the process of star formation. However, the separation distribution of PMS binaries peaks at a distance corresponding to subarcsecond angular resolution for most of the star forming regions (SFR) currently observable. Apart from some rare previous works (see e.g. Brandner \& Zinnecker 1997; Ghez et al. 1997), the basic physical data are still lacking for most of the individual members of these binary systems because of the limited angular resolution of the available instruments (cameras,

Send offprint requests to: J.-L. Monin, e-mail: Jean-Louis. Monin@obs.ujf-grenoble.fr

* Based on observations made at ESO $3.60 \mathrm{~m}$ Telescope. spectrographs and polarimeters), or the limited signal-tonoise ratio of the observations.

On the other hand, TTauri stars present a complex circumstellar environment, with possible near-infrared and visible/ultraviolet excesses, the former coming from a circumstellar disk and the latter from the related boundary layer, or hot accretion spots. Between these two borders, the bulk of the underlying star emission peaks around $1 \mu \mathrm{m}$, making this wavelength range a preferential target to estimate the actual underlying stellar luminosity (see e.g. Hartigan et al. 1994).

The recent availability of near infrared cameras coupled to adaptive optics (AO), allowing one to obtain images with a resolution down to $\approx 0.2$ arcsec or better in the range 1 to $5 \mu \mathrm{m}(J, H, K, L \& M$ bands), together with a field of view of the order of $10 \operatorname{arcsec}$ on the ESO $3.60 \mathrm{~m}$ telescope provides a unique opportunity to study the binarity and measure the physical parameters of southern TTS. In practice, binaries with linear projected distance from $1500 \mathrm{AU}$ down to $30 \mathrm{AU}$ at the Rho Ophiucus cloud distance (assumed $140 \mathrm{pc}$ ) can be resolved and imaged with a high signal to noise ratio. At such a distance, images can be obtained of binaries with companion stars 
separated by less than the canonical predicted size of an accretion disk ( $\approx 100 \mathrm{AU})$. With such a spatial resolution, the 2 to $5 \mu \mathrm{m}$ spectral range ("thermal near infrared", in the $K, L \& M$ bands) can give access to the infrared excess of both objects. This allows us to study the distribution of circumstellar material in close binaries and therefore, to address the question of the presence of circumstellar disks in close binary systems, as well as their further evolution. We can thereby classify the components as Classical T Tauri stars (CTTS, with disks), or Weak TTS (WTTS, without disk).

Prato \& Simon (1997) have shown that in PMS binaries the disks of the two components coevolve, and used arguments based on the survival time of circumstellar disks in a binary environnement to conclude that a common cause (a surrounding circumbinary environment) might be responsible for the feeding of the individual circumstellar disk. Once the external reservoir is empty, a quick dissipation of both circumstellar disks occurs. More recently, Monin et al. (1998), Duchêne et al. (1999), and White \& Ghez (1998) have performed a study of the relative accretion activity between the circumprimary and circumsecondary disks in young binary systems in Taurus, using different approaches (spectroscopy and NIR photometry). These authors independently find that in most cases, the primary accretes more than its companion, suggesting that the primary disk is longer lived or either more massive and/or preferentially replenished.

In this paper, we report the results of a study of southern PMS binaries using adaptive optics and a $1-5 \mu \mathrm{m}$ camera. We give a description of the observed sample in Sect. 2. The specific reduction procedure is presented in Sect. 3, and the results in Sect. 4. Section 5 provides a discussion on the scientific implications of our results, and our work is summarised in Sect. 6 .

\section{Observations}

\subsection{Objects selection}

A sample of a dozen southern PMS binaries was chosen in Ophiuchus, Lupus, TW Hya and Chamaeleon clouds from the list of Reipurth \& Zinnecker (1993), using the following criteria: separation range of 40-400 AU (median value $100 \mathrm{AU}$ ) so that we could measure the NIR photometry of previously unresolved companions; infrared colors allowing proper measurements in the $3-5 \mu \mathrm{m}$ range, and objects bright enough to allow good AO correction ( $V \leq 14 \mathrm{mag}$ ). The observed binaries, together with their characteristics extracted from the literature, are listed in Table 1.

For some of the sources listed in Table 1, we could estimate from the literature the extinction coefficient $A_{V}$ using the color index $\left(R_{\mathrm{C}}-I_{\mathrm{C}}\right)$ and the spectral type of the primary, assuming that this index is less subject to UV or IR excess contamination and that the intrinsic $\left(R_{\mathrm{C}}-I_{\mathrm{C}}\right)_{\mathrm{o}}$ colors of $\mathrm{T}$ Tauri stars are similar to main sequence dwarfs (Hartigan et al. 1994). Moreover, we adopted the extinction law of Rieke \& Lebofsky (1985), so that $A_{V}=3.76 E\left(R_{\mathrm{C}}-I_{\mathrm{C}}\right)$. The resulting values are given in Table 1 . We also give there the classification of each binary as a whole as CTTS or WTTS, using the criterion of Martín (1998), with an $\mathrm{H} \alpha$ equivalent width threshold varying with the spectral type; hence we classify the stars depending on their $\mathrm{H} \alpha$ flux.

\subsection{Observations with ADONIS/SHARP \& COMIC}

The image correction was performed by the ADONIS facility (see Beuzit et al. 1997 for further details). The average atmospheric conditions as estimated in the visible by the ADONIS system are summarized hereafter: seeing $\simeq 0^{\prime \prime} 8$, turbulence coherence time $\tau_{0} \simeq 300 \mathrm{~ms}$, and Fried parameter $r_{0} \simeq 15-25 \mathrm{~cm}$.

From the adaptive optics point of view, TTS represent a tough challenge for the correction system since they are often dimmer than $V=12 \mathrm{mag}$ and most interesting objects are often found around $V=14 \mathrm{mag}$. The wavefront sensor detector performed excellently and we were able to close the loop on the primary (i.e. brighter at $V$ ) component of all our sources, even one reported as faint as $V=14.9 \mathrm{mag}$ in the literature (V 536 Aql, Herbig \& Bell 1988); however, TTS are known to vary by several magnitudes and this $V=14.9$ mag value should not be used to assess the limiting performances of the ADONIS instrument. Every source was observed using the primary as a reference star. This solution worked correctly when there was sufficient magnitude difference $\Delta m$ between the primary and its companion, and when the binary separation was not too large (typically $<1$ ". 5 for a wavefront sensor pixel scale of $\simeq 0$.' 85 on the sky). When both components of the binary were of the same brightness, image degradation was observed. In such case, the use of a numerical filter masking one of the components successfully recovered the correction capabilities of the instrument. The sky background was measured and subtracted every few seconds with a chopping throw of $20^{\prime \prime}$, using a chopping mirror installed on the optical bench of ADONIS.

1-2.5 $\mu \mathrm{m}$ observations were performed using the SHARP camera (Hofmann et al. 1995) during two nights (1996, May 1 and 2). The new COMIC camera (Lacombe et al. 1998) was used during the last night of the run (1996, May 5) and allowed observations in $L$ and $M$ bands. These were the first scientific observations performed with the COMIC instrument (Monin \& Geoffray 1997). The weather was excellent and stable during the whole run and the instrument did run smoothly thanks to the help of the ADONIS \& SHARP/COMIC team. The pixel scale on the IR camera was 35 mas in $J H \& K$ bands and 40 mas in $L \& M$ bands. A Point Spread Function (PSF) reference star was observed before and after each scientific object, no more than 5 minutes sooner or later than the source on average, to allow further deconvolution and PSF fitting. Photometric calibrators were also observed every hour or 
Table 1. Observed sample; data from the literature, unless noted with a "*». When some parameters were already known for the companion, we have added a second line for the corresponding object

\begin{tabular}{|c|c|c|c|c|c|c|c|c|c|c|c|c|c|}
\hline Object & $\begin{array}{l}\text { sep. } \\
\left({ }^{\prime \prime}\right)\end{array}$ & $\begin{array}{l}\text { PA } \\
\left({ }^{\circ}\right)\end{array}$ & ref. & cloud & $\begin{array}{l}\text { dist. } \\
\text { (pc) }\end{array}$ & ref. & Sp. type & ref. & $\begin{array}{l}E W(\mathrm{H} \alpha) \\
(\AA)\end{array}$ & ref. & $\begin{array}{l}A_{V} \\
(\mathrm{mag})\end{array}$ & ref. & $\begin{array}{l}\mathrm{C} / \mathrm{W} \\
\text { type }\end{array}$ \\
\hline \multirow[t]{2}{*}{ BK Cha } & \multirow[t]{2}{*}{0.8} & \multirow[t]{2}{*}{329} & \multirow[t]{2}{*}{1} & \multirow[t]{2}{*}{ Chamaeleon II } & \multirow[t]{2}{*}{180} & \multirow[t]{2}{*}{5} & K7-M0 & 3 & $35-114$ & $3,4,6$ & 2.2 & 6 & \multirow[t]{2}{*}{$\mathrm{C}$} \\
\hline & & & & & & & M0.5 & 6 & 29 & 6 & 1.6 & 6 & \\
\hline CD-29 8887 & 0.8 & 40 & 1 & TW Hya & 50 & 7 & M0 & 8 & 2 & 8 & 0.7 & $*$ & W \\
\hline \multirow{2}{*}{ Hen 3-600 } & \multirow[t]{2}{*}{1.4} & \multirow[t]{2}{*}{230} & \multirow[t]{2}{*}{8} & \multirow{2}{*}{ TW Hya } & \multirow[t]{2}{*}{50} & \multirow[t]{2}{*}{7} & M3 & 8 & 20 & 8 & 0.7 & $*$ & \multirow[t]{2}{*}{$\mathrm{C}$} \\
\hline & & & & & & & M3 & 8 & 8 & 8 & 0.7 & $*$ & \\
\hline \multirow[t]{2}{*}{ HO Lup } & \multirow[t]{2}{*}{1.5} & \multirow[t]{2}{*}{36} & \multirow[t]{2}{*}{1} & \multirow[t]{2}{*}{ Lupus III } & \multirow[t]{2}{*}{150} & \multirow[t]{2}{*}{10,11} & K7-M0 & 6 & \multirow[t]{2}{*}{220} & \multirow[t]{2}{*}{9} & \multirow[t]{2}{*}{1.25} & \multirow[t]{2}{*}{9} & \multirow[t]{2}{*}{$\mathrm{C}$} \\
\hline & & & & & & & M2 & 6 & & & & & \\
\hline SR 9 & 0.59 & 350 & 2 & Ophiuchus & 140 & 12 & K4-K6 & 13,14 & $11-26$ & 13 & 0.3 & $*$ & $\mathrm{C}$ \\
\hline $\mathrm{Sz} 68$ & 2.6 & 295 & 1 & Lupus I & 150 & 10,11 & $\mathrm{~K} 2$ & 15,16 & $2-7$ & $9,17,18$ & 1.45 & 9 & $\mathrm{C}$ \\
\hline V536 Aql & 0.52 & 17 & 19 & & 200 & 20 & $\mathrm{~K} 7$ & 21 & 52 & 21 & 3.3 & 21 & $\mathrm{C}$ \\
\hline V653 Oph & 0.4 & 96 & 2 & Ophiuchus & 140 & 12 & M2.5 & 13,21 & $30-48$ & 13,21 & 2 & $*$ & $\mathrm{C}$ \\
\hline WSB 20 & 1 & 23 & 1 & Ophiuchus & 140 & 12 & K6 & 21 & 47 & 21 & 2.3 & $*$ & $\mathrm{C}$ \\
\hline НBC634 & 0.2 & 164 & 2 & Scorpio & 160 & 22 & K0 & 23 & 0.35 & 23 & 1.3 & 23 & W \\
\hline HD76534 & 2.1 & 303 & 1 & DC164.3+1.5 & 830 & 24,26 & $\mathrm{~B} 2$ & 25 & & & 1.2 & $*$ & $\mathrm{HAe}$ \\
\hline
\end{tabular}

$\left(^{*}\right)$ this study, see text; (1) Reipurth \& Zinnecker 1993; (2) Ghez et al. 1993; (3) Hughes \& Hartigan 1992; (4) Hartigan et al. 1993; (5) Wittet et al. 1997; (6) Brandner \& Zinnecker 1997; (7) Kastner et al. 1997; (8) De La Reza et al. 1989; (9) Hughes et al. 1994; (10) Hughes et al. 1993; (11) Franco 1990; (12) De Geus \& Burton 1991; (13) Bouvier \& Appenzeller 1992; (14) Herbig \& Bell 1988; (15) Franchini et al. 1992; (16) Appenzeller et al. 1983; (17) Herbst et al. 1994; (18) Bouvier 1990; (19) Ageorges et al. 1994; (20) Kholopov 1959; (21) Cohen \& Kuhi 1979; (22) De Geus et al. 1989; (23) Walter et al. 1994; (24) Van Den Ancker et al. 1998; (25) Finkenzeller 1985; (26) Herbst 1975.

so, and our observations were calibrated using the Bessell \& Brett (1988) standard system.

All of our targets were observed with high photometric signal/noise ratio, typically 500 in $J, H \& K$ bands, and $>100$ in $L \& M$ bands. The large integration capacity of the COMIC detector allowed individual frame integration times as high as $10 \mathrm{~s}$ in $L$ band and $1 \mathrm{~s}$ in $M$ band. In each case, the integration time was adjusted to get approximately half of the saturation level in the detector. In this configuration, the readout noise is dominated by the sky background statistical noise. Such long integration times are not essential from a signal-to-noise point of view but are very convenient to increase the acquisition efficiency (integration time compared to total observing time), which can be a concern during NIR observations. Objects as faint as $L=10.5 \mathrm{mag}$ and $M=9.2 \mathrm{mag}$ have been observed with total integration times of $180 \mathrm{~s}$ and $230 \mathrm{~s}$ respectively. Some faint objects observed in $J H K$ bands could not be observed in $L$ and $M$ bands.

\section{Data reduction}

\subsection{Near Infrared background subtraction}

Data reduction was performed using the ECLIPSE package developed by N. Devillard at ESO (Devillard 1997), GRAPHIC and CLASS (two software packages developed at the Grenoble Observatory), and IRAF. Some hints for high background COMIC data reduction have been presented in Monin \& Geoffray (1997), and we summarize their key results in the following.
In $J, H$ and $K$ band the sky subtraction residuals are typically of $0.5 \mathrm{ADU} /$ pixel, and can introduce a photometric error for the faintest objects. Aperture photometry was performed in order to minimize the influence of this residual on photometric measurements. On the other hand, one of the main concerns of $3-5 \mu \mathrm{m}$ NIR observations is the sky background estimation and subtraction. The ADONIS bench is equipped with a tilt mirror to measure the sky emission on a nearby position. It is well known in thermal infrared imaging that the use of a chopping mirror, together with a nodding technique, allows one to remove the background from the images, both of sky and instrumental origin. However, the ADONIS bench has been mainly designed for visible and "optical" near infrared $(1-2.5 \mu \mathrm{m})$ observations, and beam-switching is not currently available. In our $L \& M$ observations, we only used the ADONIS chopping facility and estimated the residual background a posteriori in every image. This was done by fitting a polynomial baseline on every line of each frame. We found this method more efficient than trying to fit a 2-D surface on the overall image. In any case, this implies having to define a part of the line where the fit procedure must ignore the signal, as is regularly done, for instance in radioastronomy. The positions of both components of the binaries were estimated from the literature or from our previous $J H K$ images, and the size of the ignored window was chosen beyond the first diffraction ring. During the $3-5 \mu \mathrm{m}$ observing night, the seing in the $L \& M$ bands was of the order of $0.5^{\prime \prime}$, only about twice the diffraction limit, and the Strehl ratio reached values as high as $80 \%$ in $M$, so that the residual seeing halo 
contribution outside the blocking window was considered as negligible (see Fig. 3 in Monin \& Geoffray 1997).

Despite the strong background, this procedure proved to give good results, with of course smaller $S / N$ ratios than in the $1-2.5 \mu \mathrm{m}$ bands. The main limitations come from some residuals after sky subtraction due to background inhomogeneities in the AO bench. We were also limited by the fact that these inhomogeneities are modulated by the AO correction process. The study of this latter, somewhat subtle effect, is beyond of the scope of this paper and will be detailed in a forthcoming paper (Geoffray et al. 2001).

\subsection{Subsequent image processing}

A regular image processing, including bad pixels correction and flat fielding, was then applied to our backgroundsubtracted images.

To estimate the individual magnitudes of both components in our objects, we measured the integrated flux on the system and used a deconvolution algorithm to derive the flux ratio of the two components. From our data and using comparison with simulated binaries, the LucyRichardson algorithm proved to give the best results. As our sources are quite well-separated pointlike sources, we typically used about a hundred iterations, until the flux values converged. Before each deconvolution, the primary was centered on a pixel, but no subpixel resampling was applied. When the deconvolution converged, almost all the flux was concentrated in a single pixel, and the flux ratio between the two components was determined from the peak heights in the deconvolved images.

As an example, Fig. 1 shows the images obtained on the object V536 Aql, together with the associated PSF and the resulting deconvolved image (convolved with a gaussian of $F W H M$ corresponding to the one obtained on the raw images, for aesthetic purpose).

\section{Results}

\subsection{Photometric results}

The near infrared magnitudes of the individual binary components are given in Table 2. Our measurements were evaluated in the standard photometric system established by Bessell \& Brett (1988). The photometric errors on the primaries are typically $0.02-0.03 \mathrm{mag}$ in the $J, H \& K$ bands, and 0.05-0.1 mag in the $L$ and $M$ bands respectively; these uncertainties can increase by about $30-50 \%$ for the fainter secondaries. The main sources of error are sky subtraction uncertainty, flux ratio evaluation variations and to a minor extent color transformation uncertainties from the natural system of the instrument to the standard system. Some companions could not be detected in all $1-5 \mu \mathrm{m}$ bands. This is the case for HBC 634 (sep $0.2^{\prime \prime}$ ) in the $J$ band where the two components could not be resolved, or for some of the faintest objects of our list.

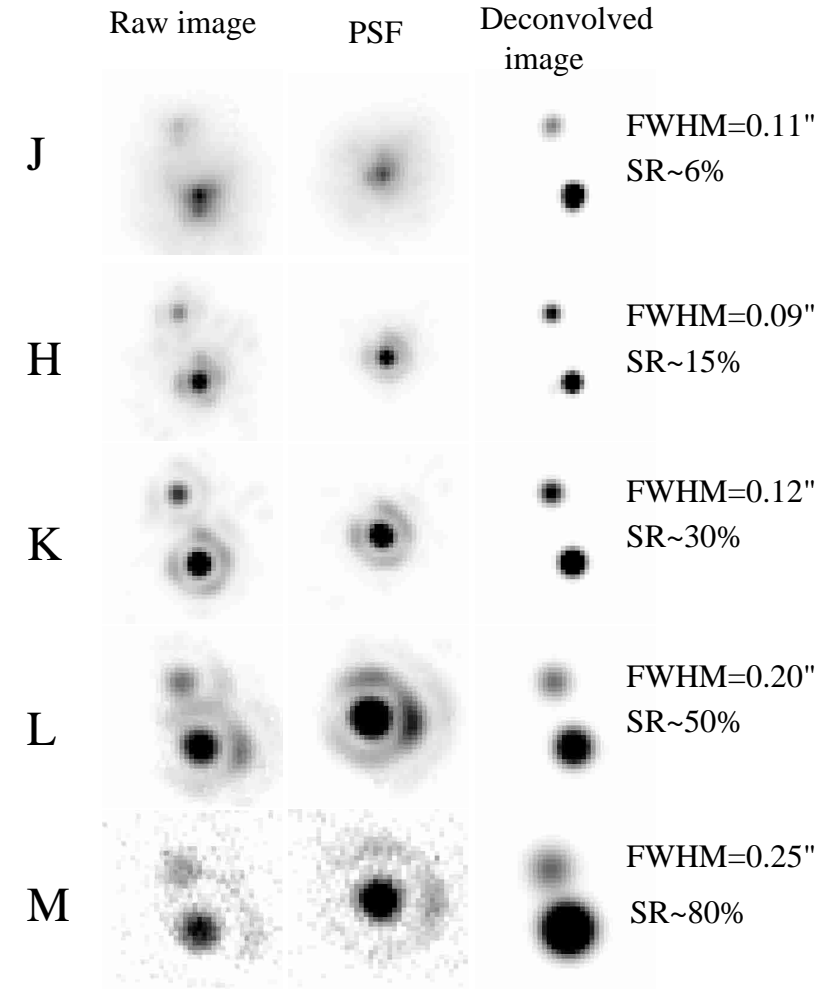

Fig. 1. Images of V536 Aql observed between 1 to $5 \mu \mathrm{m}$ ( $J H K L M$ bands). Sep $=0 . \prime 54, \mathrm{PA}=17^{\circ}$. North is up and East is to the left. The pixel scale is 35 mas in the $J, H \& K$ bands, and 40 mas in the $L \& M$ bands, so that the binary separation in pixels is smaller in the $L \& M$ bands than in the $J, H, \& K$ bands, and there is no real separation variation from 1 to $5 \mu \mathrm{m}$. Full Width at Half Maximum (FWHM) and Strehl Ratio (SR) of the PSF are given for each observing band

\subsection{Color-color diagrams and TTS classification}

Our objects have been placed in a $J-H / H-K$ colorcolor diagram (Fig. 2). The diagram also shows the Main Sequence dwarfs \& giants locus, the CTTS locus (Meyer et al. 1997) and a reddening vector corresponding to $A_{V}=5$. For most of the sources, we can estimate a value for the interstellar extinction and the spectral type for stars later than K7-M0 (after the breakpoint in the main sequence dwarf locus on the $J-H / H-K$ diagram), by dereddening the stars back along the reddening vector. The values we obtain are consistent with previous estimates from the literature. V536 Aql-B and V853 Oph$\mathrm{B}$ are exceptions within this framework and appear to be heavily extincted objects, or with a different $A_{V}$ on the primary than on the secondary. Both objects are twin CTTS/CTTS and the $A_{V}$ difference could be due to differences in the inclination of the circumstellar disks of the primary and the secondary.

Some objects deserve more comment.

HD $\mathbf{7 6 5 3 4}$ is an Herbig Be star with a possible IR excess on the primary, while the secondary lies on the Main Sequence. Bouvier \& Corporon (2000) have published a study on HAeBe stars with companions and suggested that the lack of IR excess on the secondary might be 
Table 2. Photometric results obtained for the objects of our sample in $J, H, K, L$ and $M$ bands

\begin{tabular}{lcccccc}
\hline \hline Object & & $J$ & $H$ & $K$ & $L$ & $M$ \\
\hline \hline \multirow{3}{*}{ BK Cha } & A & 11.41 & 10.36 & 9.65 & 8.63 & \\
& B & 11.49 & 10.22 & 9.26 & 7.98 & \\
CD-298887 & A & 8.03 & 7.31 & 7.12 & 7.04 & 6.8 \\
& B & 8.87 & 8.15 & 7.93 & 7.83 & \\
HD 76534 & A & 8.06 & 7.99 & 7.86 & 7.61 & 7.7 \\
& B & 9.38 & 9.37 & 9.34 & 9.34 & 9.15 \\
Hen 3-600 & A & 8.25 & 7.79 & 7.39 & 7.05 & 6.8 \\
& B & 8.69 & 8.19 & 7.82 & 7.60 & \\
HO Lup & A & 9.97 & & 8.60 & 8.03 & 7.8 \\
& B & 11.71 & & 10.59 & 10.45 & \\
SR9 & A & 8.55 & 7.84 & 7.55 & 6.79 & 6.2 \\
& B & 11.03 & 10.34 & 10.05 & 10.17 & \\
Sz68 & A & 7.77 & 7.07 & 6.69 & 6.07 & 5.4 \\
V536Aql & B & 11.13 & 10.18 & 9.47 & 8.81 & 8.7 \\
& A & 9.19 & 8.33 & 7.64 & 6.77 & 5.7 \\
V853Oph & B & 10.89 & 9.38 & 8.71 & 7.94 & 6.3 \\
& A & 9.37 & 8.66 & 8.21 & & \\
WSB20 & B & 11.37 & 10.27 & 9.77 & & \\
& A & 9.34 & 8.39 & 7.76 & 7.20 & \\
HBC 634 & B & 10.29 & 9.38 & 8.95 & 8.70 & \\
& A & & 7.88 & 7.71 & & \\
\hline The $J$ band & B & & 9.41 & 9.27 & & \\
\hline
\end{tabular}

* The $J$ band photometry of HBC634 is for the unresolved system (see text for details).

explained by the influence of the luminous primary on the circumstellar environment of the secondary. However, this star was observed during the course of this study but is not a high priority target in our paper and we do not discuss its case hereafter.

Hen 3-600 appears to be a WTTS/WTTS binary falling slightly off the main sequence on the $\mathrm{M}$ spectral type side ( $2 \sigma$ from M3 spectral type).

HBC 634 is peculiar as it is the closest binary of our sample $\left(0.2^{\prime \prime}\right)$ and its components could not properly be separated in the $J$ band, where the correction, and thus the Strehl ratio and the angular resolution provided by the AO system is worse. Hence, the $J=8.18$ magnitude given in Table 2 is an integrated magnitude. However, in order to give a rough estimate of its position in the $J-H / H-K$ diagram, we assumed that the $\Delta J$ difference between the primary and the secondary is at least of the same order in this band than in $H$ and $K$ (where both $\Delta m$ are similar). Doing so, the HBC 634 system appears to be another WTTS/WTTS pair, with a primary spectral type consistent with the K0 value given by Walter et al. (1994).

HO Lup could not be measured in the $H$ band.

In order to more confidently distinguish between stars with and without optically thick circumstellar disks, we plotted the $K-L$ indexes in Fig. 3 for both components when available. Following Edwards et al. (1993), we use the canonical value $K-L \geq 0.4$ to discriminate between the sources with or without disks (dashed lines on the plot). The general trend in Fig. 3 is a correlation between

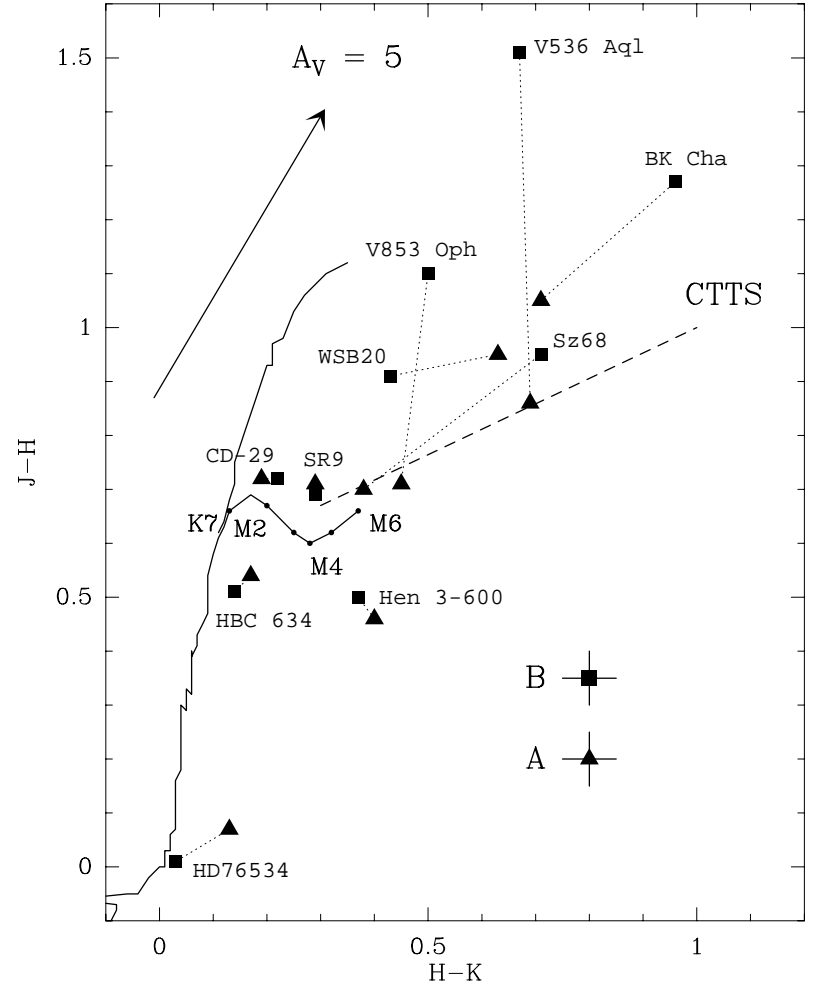

Fig. 2. $J-H / H-K$ color-color diagram; primaries are plotted as full triangles, secondaries as full squares; the cross on the A/B symbols gives the one sigma uncertainty of the measurements. The CTTS locus (Meyer et al. 1997) is plotted as a dashed line and a reddening vector corresponding to $A_{V}=5$ has also been plotted. The label of CD-29 8887 has been abreviated as CD-29 for the sake of clarity, in this figure and in the following

the $K-L$ indexes in both components. Given the photometric uncertainties, WSB 20 and HO Lup are close to the $(0.4,0.4)$ crossing point. The position of the SR9 point is somewhat surprising, as the $J-H / H-K$ diagram shows this source to be close to a WTTS/WTTS twin, with a spectral type close to M2-M4. However, it is also close to the beginning of the CTTS locus, and the primary could harbour a small infrared excess. On the other hand, we cannot discard the possibility of a measurement error on this source. Apart from SR9, this correlation reflects the twinning tendency of PMS binaries (see e.g. Prato \& Simon 1997; Duchêne et al. 1999 and references therein): when a component in a resolved binary is of a given type (C or W-TTS), most of the time, so is the other. None of the previously unresolved WTTS systems shows high $K-L$ values, in other words both components are WTTS. For previously unresolved CTTS systems, half of them correspond to CTTS/CTTS pairs, the other half being charaterized by a CTTS primary and a WTTS companion. In other words, in all the mixed systems of our sample, the primary still has a disk while the secondary disk is already gone. The detailed classification of the components of the pairs of our sample are sumarized in Table 3. For the sake of simplicity, the CTTS or WTTS pairing were summarized respectively as CC, CW \& WW. 


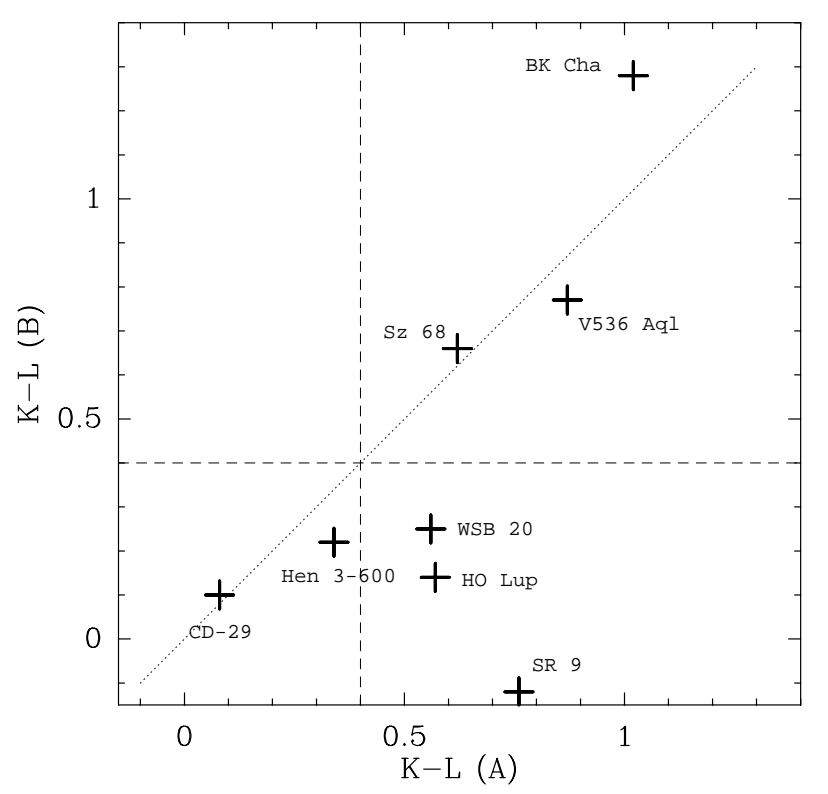

Fig. 3. $(K-L)$ index of the secondary as a function of the index of the primary for the measured TTS binary systems in our sample. We have used $A_{V}$ estimations from the literature to apply a dereddening correction to our sources. However, due to the relatively low absorption present in our sources and the interstellar extinction at $K$ and $L$, this correction was always small. Sources labelled as in Fig. 2

Table 3. Components classification as Classical or Weak TTS in the binaries of our sample

\begin{tabular}{lll}
\hline \hline Objet & Type & Sep(AU) \\
\hline \hline BK Cha & CC & 140 \\
CD-29 8887 & WW & 40 \\
Hen 3-600 & WW & 60 \\
HO Lup & CW & 225 \\
SR9 & CW & 80 \\
Sz68 & CC & 390 \\
V536Aql & CC & 100 \\
V853Oph & CC & 60 \\
WSB20 & CW & 140 \\
HBC 634 & WW & 33 \\
\hline
\end{tabular}

Two of our sources lack L photometry. However, we can still use the available measurements to tentatively classify them as C or W-TTS. This is the case for V853 Oph which we classify as a twin CTTS/CTTS binary on the basis of its position in the $J-H / H-K$ diagram. HBC 634 has been discussed previously. We therefore get a sample of 10 binaries with $\mathrm{C} / \mathrm{W}$ pairing information (not including HD 76534). Prato \& Monin (2000) have reviewed spectral accretion diagnosis in PMS binaries and they find that among 29 southern binary systems, between 25 and $35 \%$ of the systems are mixed, depending on the correction they apply. In our study, although based on a smaller sample, we find that among 10 systems, 3 are mixed, therefore our result is entirely consistent with previous measurements.
A new interesting result of this classification of close binaries is that all the 3 WTTS/WTTS pairs of our sample have separations less than 60 AU. Although once again based on small numbers, this effect, if real, could result from faster disk dispersal in close binaries due to strong tidal interactions between both components.

\subsection{SED fits}

The availability of our new $J H K L M$ photometry for both components in close binary systems where previous unresolved visible photometry was known, allows us to go further in the study of the physical parameters of our sources. For 7 binary systems out of 10 (not including HD 76534), a fit of the spectral energy distribution of each component of the binary systems was performed. The spectral energy distributions corrected for interstellar extinction were fitted with standard intrinsic colors of MS dwarfs, assuming the extinction is identical on both components. The free parameters of the fit are the spectral type of the primary, the visual magnitude and the spectral type of the secondary. When the flux ratio of the binary is high enough in the visible, we assume that the spectral type of the primary is the same as the one measured for the unresolved system. In our results the spectral type for each component is constrained to within one subclass. The errors on both the spectral type and the $V$ magnitudes mainly come from the uncertainty on the interstellar extinction coefficient $A_{V}$, from the photometric variability of the objects (the photometric measurements are not simultaneous) and, to a minor extent, to photometric uncertainties.

We give hereafter some comments on the results of the fits presented in Fig. 4.

- BkCha: VI photometry, spectral type and $A_{V}$ are all known from the literature (references from Table 1 ). The fit is therefore straightforward on the visible part of the spectrum. Both components show high infrared excess, as can already be seen in the $J-H / H-K$ diagram;

- CD-29 8887: both components are WTTS. The fit is well constrained by $J H K L M$ photometry on each component;

- Hen 3-600: idem as the previous object;

- HO Lup: the flux ratio of the binary is large in the visible and we choose the spectral type of the primary to be the spectral type of the unresolved system. The secondary appears to be a WTTS without any infrared excess and we adjust its visual magnitude and its spectral type;

- SR9: idem as the previous object;

- Sz68: RI photometry for each component is known from the literature. As the flux ratio is large in the visible, we keep the spectral type of the unresolved object for the primary. The secondary has low infrared excess in $J$ band, which allows us to fit its spectral type and visual magnitude on the $R I J$ photometry;

- WSB20: same as HO Lup and SR9. 

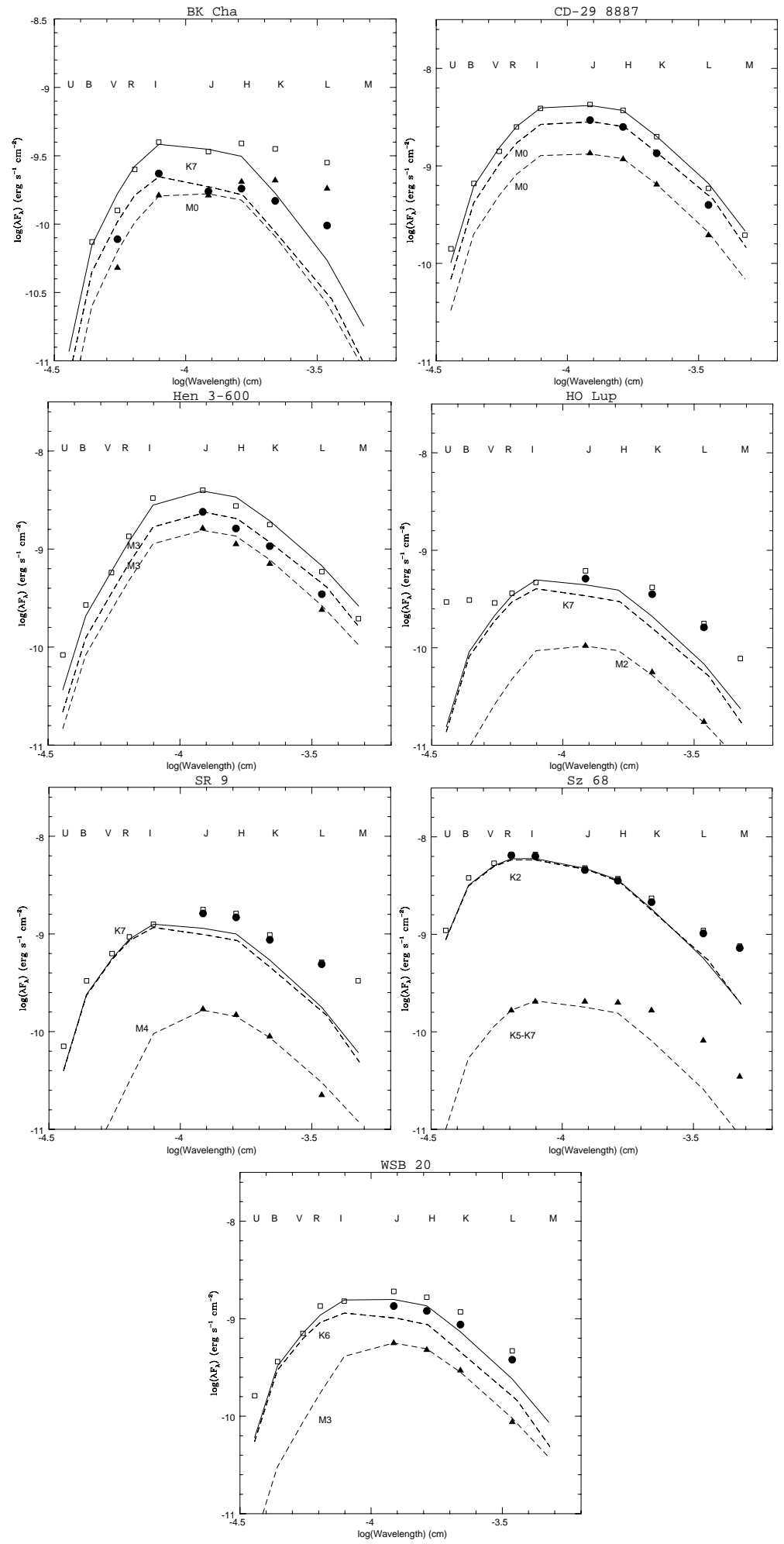

Fig. 4. SED fits. Primaries: filled circles, secondaries: filled triangles. The integrated flux is represented by empty squares

\subsection{HR diagram}

Knowing the temperature from the spectral type and the luminosity from the visual magnitude of the objects, we placed them in an H-R diagram (see Fig. 5), where the evolutionary tracks computed by D'Antona \& Mazzitelli
(1994, hereafter DM94) have also been plotted. From this diagram, we estimate the physical parameters for both components of the binaries in our sample. Uncertainties on these estimations are within a factor of two on the age and $\approx 0.1 M_{\odot}$ on the mass. In order to obtain more confidence in the physical parameters of our objects, we have 
Table 4. Physical parameters for both components of the 7 fitted binaries

\begin{tabular}{|c|c|c|c|c|c|c|c|c|c|}
\hline \multirow[b]{2}{*}{ Objet } & & \multirow[b]{2}{*}{$\log \left(T_{\text {eff }}\right)$} & \multirow[b]{2}{*}{$\log \left(L / L_{\odot}\right)$} & \multicolumn{3}{|c|}{ DM94 } & \multicolumn{3}{|c|}{ PS99 } \\
\hline & & & & Age (yr) & $M\left(M_{\odot}\right)$ & $q$ & Age (yr) & $M\left(M_{\odot}\right)$ & $q$ \\
\hline \multirow[t]{2}{*}{ BK Cha } & $\mathrm{A}$ & 3.60 & -0.49 & $510^{6}$ & 0.70 & \multirow{2}{*}{0.78} & $510^{6}$ & 0.70 & \multirow{2}{*}{0.85} \\
\hline & B & 3.58 & -0.62 & $410^{6}$ & 0.55 & & $510^{6}$ & 0.60 & \\
\hline \multirow[t]{2}{*}{ CD-29 8887} & $\mathrm{~A}$ & 3.58 & -0.49 & $210^{6}$ & 0.50 & \multirow{2}{*}{1.2} & $310^{6}$ & 0.60 & \multirow{2}{*}{1.0} \\
\hline & B & 3.58 & -0.82 & $110^{7}$ & 0.60 & & $110^{7}$ & 0.60 & \\
\hline \multirow[t]{2}{*}{ Hen 3-600 } & A & 3.53 & -0.64 & $110^{6}$ & 0.22 & \multirow{2}{*}{1.0} & $110^{6}$ & 0.25 & \multirow{2}{*}{1.0} \\
\hline & B & 3.53 & -0.82 & $210^{6}$ & 0.22 & & $210^{6}$ & 0.25 & \\
\hline \multirow[t]{2}{*}{ HO Lup } & $\mathrm{A}$ & 3.60 & -0.38 & $310^{6}$ & 0.60 & \multirow{2}{*}{0.5} & $510^{6}$ & 0.70 & \multirow{2}{*}{0.43} \\
\hline & B & 3.54 & -0.96 & $310^{6}$ & 0.30 & & $510^{6}$ & 0.30 & \\
\hline \multirow[t]{2}{*}{ SR 9} & A & 3.60 & 0.02 & $610^{5}$ & 0.40 & \multirow{2}{*}{0.35} & $110^{6}$ & 0.70 & \multirow{2}{*}{0.2} \\
\hline & B & 3.50 & -0.96 & $110^{6}$ & 0.14 & & $110^{6}$ & 0.15 & \\
\hline \multirow[t]{2}{*}{$\mathrm{Sz} 68$} & $\mathrm{~A}$ & 3.69 & 0.77 & $510^{5}$ & 1.30 & \multirow{2}{*}{0.62} & $110^{6}$ & 2.2 & \multirow{2}{*}{0.34} \\
\hline & B & 3.62 & -0.68 & $310^{7}$ & 0.80 & & $210^{7}$ & 0.75 & \\
\hline \multirow[t]{2}{*}{ WSB 20} & A & 3.62 & 0.13 & $610^{5}$ & 0.50 & \multirow{2}{*}{0.44} & $210^{6}$ & 1.0 & \multirow{2}{*}{0.25} \\
\hline & B & 3.53 & -0.37 & $310^{5}$ & 0.22 & & $610^{5}$ & 0.25 & \\
\hline
\end{tabular}

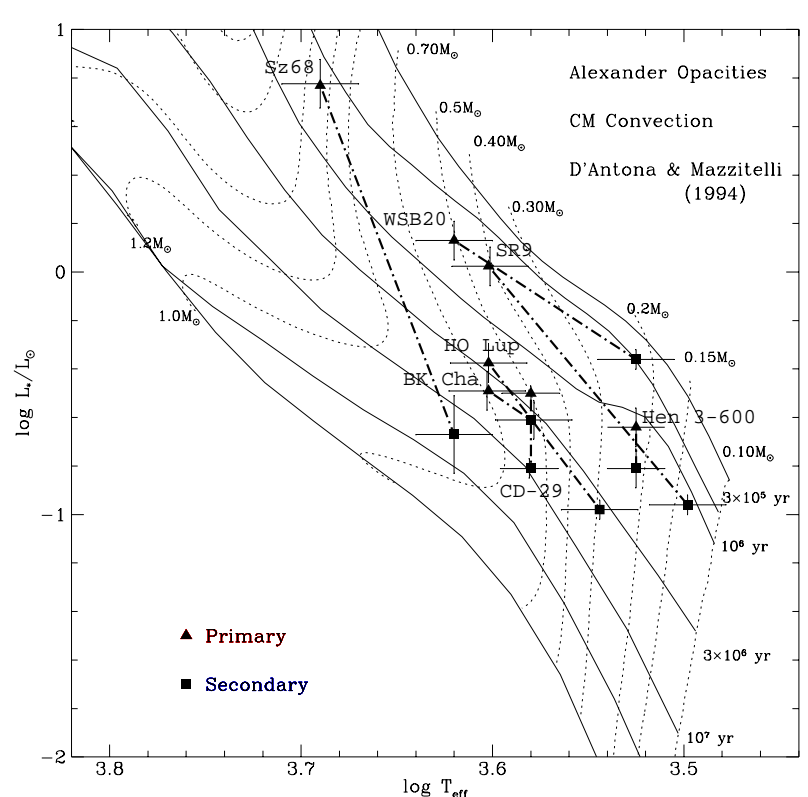

Fig. 5. HR diagram. The source labels are placed close to the primary, except for CD-29 8887

also used the tracks from the more recent computations of Palla \& Stahler (1999, hereafter PS99). Both list of extracted values are given in Table 4 . The following results can be drawn from this study (they hold whatever set of tracks used):

- Most of these systems are coeval within the errorbars (age difference less than a factor of three for BK Cha, Hen 3-600, HO Lup, Sr 9 and WSB 20). Their separations range from 60 to $220 \mathrm{AU}$;

- CD-298887 components are marginally coeval, within a factor of 3-5, depending on the tracks used;

- Sz68 is probably not coeval. Note that $\mathrm{Sz} 68$ primary is itself a binary with $0.1^{\prime \prime}$ separation and a flux ratio of 6
(Ghez et al. 1997b); lowering the primary's luminosity brings the two components a bit closer to co-evality but not significantly. However, Sz68 is the widest pair of our sample and might not be a true binary.

Hartigan et al. (1994) studied binaries in Taurus with separations ranging from 400 to $6000 \mathrm{AU}$. They found that $2 / 3$ of the binaries of their sample are coeval. More recently Brandner \& Zinnecker (1997) studied 14 binary systems (in Chamaeleon, Lupus, and $\rho$ Ophiuchus clouds) with separations ranging from 90 to $250 \mathrm{AU}$ and found that for all pairs the individual components appear to be coeval. The results presented here are in agreement with these previous studies.

Finally, if we extract the mass ratios from the $\mathrm{H}-\mathrm{R}$ diagram in Fig. 5, we find that all the mixed systems have more extreme mass ratios $\left(q=M_{2} / M_{1}<0.5\right)$, while mass ratios more closer to unity $(q \geq 0.8)$ are associated with twin binaries, with the only exception of Sz68, which may not be a true binary.

\section{Discussion}

Although based on small numbers, we can briefly discuss these results within the framework of current models of binary formation and evolution.

We find a (small) proportion of mixed systems, consistent with previous estimates in southern SFR (Prato \& Monin 2000). This small proportion of mixed systems has been argued to support the idea that a circumbinary disk is the common cause of co-evolution of the two disks around the binary components. None of our adaptive optics images shows evidence of a circumbinary environment; however, our images may not possess a high enough dynamic range to allow detection of such a circumbinary environment. 
In all the mixed systems we find, the primary always harbours the active accretion disk (all our mixed systems are $\mathrm{C} / \mathrm{W}$, not $\mathrm{W} / \mathrm{C}-\mathrm{TTS}$ ). If disk replenishment proceeds from a circumbinary environment, models from Bate \& Bonnell (1997) show that the infalling material has a low angular momentum, i.e. this material falls toward the center of mass of the system, close to the primary's position.

More recently, Armitage et al. (1999) have studied the evolution of disks in close binary systems. They find that the secondary's disk should always become weaker than that surrounding the primary at a sufficiently late epoch but that the timing of the switchover is a strong function of the mass ratio of the binary. In binaries with mass ratio $<0.5$, the faster evolution of the smaller disk could dominate over the influence of the initial conditions so that the proportion of mixed systems should increase. Our observations support this prediction, as all the mixed systems of our sample have mass ratios below 0.5 . This result holds whatever set of tracks (DM94 or PS99) we use.

Finally, we note that all the WTTS/WTTS systems are found in the closest binaries of our sample $(<60 \mathrm{AU})$. As these binaries are not significantly older than other pairs, this result supports the idea that the reduced viscous time $t_{\nu} \approx R_{\text {out }}^{2} / \nu_{\text {out }}$ at the outer edge leads to faster disk evolution in these close systems.

\section{Summary}

We used $(1-5 \mu \mathrm{m})$ infrared cameras coupled with an adaptive optics system to measure high spatial resolution photometry on a sample of ten southern close PMS TTS binaries. For most of the objects of our sample, we present for the first time NIR measurements longward of $3 \mu \mathrm{m}$ for both components of each binary. We have used our measurements to place the binary components in a $J-H / H-K$ color-color diagram where we can differentiate the contribution of interstellar extinction from their intrinsic reddening. The use of the $K-L$ color index allows us to assess the presence of warm circumstellar material around each component and thus separate WTTS from CTTS. Finally, we fit the SEDs of 7 of our objects to derive their stellar properties.

The study of the pairing within the 10 southern binaries surveyed shows no significant difference with binaries from other southern clouds (Prato \& Monin 2000). In all the mixed binaries we found ( $30 \%$ of the sample), the primary is always the Classical component. If accretion proceeds from a circumbinary environment, this shows that the infalling material has low angular material (Bate \& Bonnell 1997). We find that the mixed systems have more extreme mass ratios than twin ones, a result consistent with the theoretical prediction of Armitage et al. (1999). All the WTTS/WTTS systems are found in close $(<60 \mathrm{AU})$ binaries, a result consistent with a reduction of the viscous time at the outer edge of the disk due to tidal disk truncation. Apart from one of the widest systems of our sample (Sz68, $390 \mathrm{AU})$, all the systems in our sample appear to be coeval.
Acknowledgements. We warmly thank the ESO ADONIS and COMIC/SHARP team for their invaluable help and advice during the observations. This research made use of the Simbad database, operated at CDS, Strasbourg, France. H.G. acknowledges the allocation of an ESO studentship grant during 1996/97. We thank the referee, Wolfgang Brandner, for an extremely prompt report that helped to increase the quality of the paper.

\section{References}

Ageorges, N., Menard, F., Monin, J.-L., \& Eckart, A. 1994, A\&A, 283, L5

Appenzeller, I., Krautter, J., \& Jankovics, I. 1983, A\&AS, 53, 291

Armitage, P. J., Clarke, C. J., \& Tout, C. A. 1999, MNRAS, 304,425

Bate, M. R., \& Bonnell, I. A. 1997, MNRAS, 285, 33

Bessell, M. S., \& Brett, J. M. 1988, PASP, 100, 1134

Beuzit, J.-L., Demailly, L., Gendron, E., et al. 1997, Exper. Astron., 7, 285

Bouvier, J. 1990, AJ, 99, 946

Bouvier, J., \& Appenzeller, I. 1992, A\&AS, 92, 481

Bouvier, J., \& Corporon, P. 2000, in Proceedings of the IAU Symposium 200, The Formation of Binary Stars (Potsdam, Germany, April 2000), ed. R. Mathieu, \& H. Zinnecker, in press

Brandner, W., \& Zinnecker, H. 1997, A\&A, 321, 220

Cohen, M., \& Kuhi, L. V. 1979, ApJ, 41, 743

D'Antona, F., \& Mazzitelli, I. 1994, ApJS, 90, 467

De Geus, E. J., De Zeeuw, P. T., \& Lub, J. 1989, A\&A, 216, 44

De Geus, E. J., \& Burton, W. B. 1991, 246, 559

De La Reza, R., Torres, C. A. O., \& Quast, G. 1989, ApJ, 343, L61

Devillard, N. 1997, The Messenger, 87, 19

Duchêne, G. 1999, A\&A, 341, 547

Duchêne, G., Monin, J.-L., Ménard, F., \& Bouvier, J. 1999, A\&A, 351, 954

Edwards, S., Ray, T., \& Mundt, R. 1993, in Protostars and planets III, ed. E. Levy, \& J. Lunine (The University of Arizona Press, Tucson), 567

Finkenzeller, U. 1985, A\&A, 151, 340

Franchini, M., Covino, E., Stalio, R., \& Terranegra, L. 1992, A\&A, 256, 525

Franco, G. A. P. 1990, A\&A, 227, 499

Ghez, A. M., Neugebauer, G., \& Matthews, K. 1993, AJ, 106, 2005

Ghez, A. M., White, R. J., \& Simon, M. 1997a, AJ, 490, 353

Ghez, A. M., McCarthy, D. W., Patience, J. L., \& Beck, T. L. 1997b, ApJ, 481, 378

Hartigan, P. 1993, AJ, 105, 1511

Hartigan, P., Strom, K. M., \& Strom, S. E. 1994, ApJ, 427, 961

Herbig, G. H., \& Bell, K. R. 1988, LOB, 1111, 1

Herbst, W. 1975, 80, 683

Herbst, W., Herbs, D. K., \& Grossman, E. J. 1994, AJ, 108, 1906 
Hofmann, R., Brandl, B., Eckart, A., Eisenhauer, F., \& Tacconi-garman, L. E. 1995, SPIE, 2475, 192, Highangular-resolution NIR astronomy with large arrays (SHARP I and SHARP II)

Hughes, J., \& Hartigan, P. 1992, AJ, 104, 680

Hughes, J., Hartigan, P., \& Clampitt, L. 1993, AJ, 105, 571

Hughes, J., \& Hartigan, P., Krautter, J., \& Kelemen, J. 1994, AJ, 108, 1071

Kastner, J. H., Zuckerman, B., Weintraub, D. A., \& Forveille, T. 1997, Science, 277, 67

Kholopov, P. N. 1959, SA, 3, 291

Lacombe, F., Marco, O., Geoffray, H., Beuzit, J.-L., Monin, J.-L., et al. 1998, PASP, 110, 1087

Leinert, Ch., Richichi, A., \& Haas, M. 1993, A\&A, 318, 472

Martín, E. L. 1998, AJ, 115, 351

Meyer, M. R., Calvet, N., \& Hillenbrand, L. A. 1997, AJ, 114, 288

Monin, J.-L., \& Geoffray, H. 1997, The Messenger, 89, 33
Monin, J.-L., Ménard, F., \& Duchêne, G. 1998, A\&A, 339, 113

Osterloh, M., \& Beckwith, S. V. W. 1995, ApJ, 439, 288

Palla, F., \& Stahler, S. W. 1999, ApJ, 525, 772

Prato, L., \& Simon, M. 1997, ApJ, 474, 455

Prato, L., \& Monin, J.-L. 2000, in Proceedings of the IAU Symposium 200, The Formation of Binary Stars (Potsdam, Germany, April 2000), ed. R. Mathieu, \& H. Zinnecker, in press

Rieke, G. H., \& Lebofsky, M. J. 1985, ApJ, 288, 618

Reipurth, B., \& Zinnecker, H. 1993, A\&A, 278, 81

Van Den Ancker, M. E., De Winter, D., \& Tjin A Djie, H. R. E. 1998, A\&A, 330, 145

Walter, F. M., Vrba, F. J., Mathieu, R. D., Brown, A., \& Myers, P. C. 1994, AJ, 107, 692

Whittet, D. C. B., Prusti, T., Franco, G. A. P., et al. 1997, A\&A, 327, 1194

White, R. J. \& Ghez, \& A. M. 1998, AAS, 193, 7311 